\section{Schizophrenia and cancer: an epidemiological study}

\author{
MICHAEL J. GOLDACRE, LIANNE M. KURINA, CLARE J. WOTTON, \\ DAVID YEATES and VALERIE SEAGROATT
}

\begin{abstract}
Background For decades there has been interest in the possibility that people with schizophrenia might have some protection against cancer, and that, if this were so, it might hold clues about aetiological mechanisms in schizophrenia.
\end{abstract}

Aims To study cancer incidence in schizophrenia.

\begin{abstract}
Method Cohort analysis of linked hospital and death records was used to compare cancer rates in people with schizophrenia with a reference cohort.
\end{abstract}

Results We did not find a reduced risk for cancer overall (rate ratio 0.99, 95\% Cl 0.90-1.08) or for most individual cancers. There was, however, a significantly low rate ratio for skin cancer $(0.56,95 \% \mathrm{Cl}$ 0.36-0.83).

\section{Conclusions We found no evidence} that schizophrenia confers protection against cancer in general. Low rates of skin cancer are consistent with the hypothesis that sun exposure may influence the development of schizophrenia, although other explanations are also possible.

\section{Declaration of interest None.} phrenia have a lower risk of cancer than
For nearly a century there has been interest in the possibility that people with schizothe general population, with speculation that lower susceptibility to cancer might provide clues about the biological basis of schizophrenia. Recently, several biological hypotheses have been proposed to explain why people with schizophrenia might be protected against cancer, including a protective effect of excess dopamine (Basu $\&$ Dasgupta, 2000), enhanced natural killer cell activity (Yovel et al, 2000), an increase in the rate of apoptosis (Catts \& Catts, 2000) and modulation by antipsychotic drugs of the human cytochrome enzymes involved in mutagen activation and elimination (Carrillo \& Benitez, 1999). However, the epidemiological evidence for protection is not convincing. We therefore used population-based data from a health region in the southern part of England to study the occurrence of cancer in people with schizophrenia.

\section{METHOD}

\section{Population and data}

We used data from the Oxford Record Linkage Study (Goldacre et al, 2000). This data-set includes brief statistical abstracts of records of all hospital admissions (including day cases) in National Health Service (NHS) hospitals, and all deaths regardless of where they occurred, in defined populations within the former Oxford NHS Region from 1 January 1963 to 31 March 1999. Until 1994 the data-set also included all contacts with specialist psychiatric services in the health district of Oxfordshire, including out-patients as well as day cases and in-patients. The hospital data were collected routinely in the NHS as part of the region's hospital statistics system. The death data derive from death certificates. Data collection, including those for psychiatry, covered part of one health district and its associated hospitals from 1963 (population 350000 ), two districts including Oxfordshire from 1966 (population 850000 ), six districts from 1975 (population 1.9 million) and all of the region's districts from 1987 (population 2.5 million). The data for each individual were linked together, as they accrued, as part of the region's health information system. They are now anonymised and archived.

The schizophrenia cohort was constructed by identifying the first admission to an NHS hospital during the study period, or the first contact with specialist psychiatric services for those in Oxfordshire, for each individual with the condition. A reference cohort was constructed by identifying the first admission for each individual with various medical and surgical conditions (see footnotes to Table 2). This is based on our 'reference' group of conditions, which has been used in other studies of interrelationships between diseases (Goldacre et al, 2000). We searched the database for any subsequent NHS hospital care for, or death from, cancers in these two cohorts. We considered that rates of cancer in the reference cohort would approximate those in the general population of the region while allowing for migration in and out of it (data on migration of individuals were not available).

\section{Statistical methods}

We calculated rates of each cancer based on person-years at risk. We took 'date of entry' into each cohort as the date of first admission for schizophrenia or reference condition, and 'date of exit' for the analysis of each individual cancer as the date of first record of the cancer, death or 31 March 1999, whichever was the earliest. In comparing the schizophrenia cohort with the reference cohort, we first calculated rates for each cancer, standardised by age (in 5year age groups), gender, calendar year of first recorded admission and district of residence, taking the combined schizophrenia and reference cohorts as the standard population. We then applied the overall rates to the age structure of the individual cohorts of people with schizophrenia or with the reference conditions. We calculated the ratio of the standardised rate of occurrence of cancer in the schizophrenia cohort relative to that in the reference cohort. The confidence interval for the rate ratio and $\chi^{2}$ statistics for its significance were 
calculated as described elsewhere (Breslow \& Day, 1987).

We studied the results for each condition in the reference cohort separately, as well as in combination, to ensure that no individual condition disproportionately influenced the 'expected' number of people with subsequent cancer. The people in the reference cohort who underwent appendicectomy or an operation for inguinal hernia or haemorrhoids showed significantly high subsequent admission rates for colorectal cancers. We therefore excluded these conditions from the reference cohort in the study of colorectal cancers, when comparing the schizophrenia and reference cohorts.

\section{RESULTS}

There were 9649 individuals in the schizophrenia cohort and nearly 600000 in the comparison cohort. Table 1 shows the age distribution of patients in the schizophrenia cohort at the time of first recorded admission. Some patients would already have been quite elderly by the time that data collection commenced and others would have been in long-term care. The mean age at first recorded admission for schizophrenia was 40 years and the modal age group was 25-29 years. The average length of follow-up was 12.6 years.

Table I Age distribution of study population of people admitted to hospital with schizophrenia

\begin{tabular}{lc}
\hline $\begin{array}{l}\text { Age at admission } \\
\text { (years) }\end{array}$ & $\begin{array}{c}\text { People with schizophrenia } \\
<15\end{array}$ \\
\hline $15-19$ & $140(1.4)$ \\
$20-24$ & $577(6.0)$ \\
$25-29$ & $1279(13.2)$ \\
$30-34$ & $1321(13.7)$ \\
$35-39$ & $1107(11.5)$ \\
$40-44$ & $970(10.0)$ \\
$45-49$ & $788(8.2)$ \\
$50-54$ & $702(7.3)$ \\
$55-59$ & $604(6.3)$ \\
$60-64$ & $518(5.4)$ \\
$65-69$ & $434(4.5)$ \\
$70-74$ & $409(4.2)$ \\
$75-79$ & $317(3.3)$ \\
80 and over & $234(2.4)$ \\
Total & $249(2.6)$ \\
\hline
\end{tabular}

The risk ratio for cancer, overall, in people with schizophrenia, compared with the reference cohort, was 0.99 (Table 2). The risk ratio for cancer of the lung was non-significantly raised at 1.18 and that for cancer of the oesophagus was significantly raised at 1.61 . No other individual cancer was found to be significantly more

Table 2 Occurrence of cancer in people with schizophrenia

\begin{tabular}{|c|c|c|c|c|}
\hline Cancer site or type (ICD code)' & $\begin{array}{l}\text { Cases of } \\
\text { cancer in } \\
\text { reference } \\
\text { group, }^{2} n\end{array}$ & $\begin{array}{c}\text { Observed } \\
\text { cases in } \\
\text { schizophrenia } \\
\text { cohort, } n\end{array}$ & $\begin{array}{c}\text { Expected } \\
\text { cases in } \\
\text { schizophrenia } \\
\text { cohort, } n\end{array}$ & $\begin{array}{l}\text { Adjusted rate ratio } \\
\qquad(95 \% \mathrm{Cl})\end{array}$ \\
\hline All cancer (140-208) & 26926 & 486 & 493 & $0.99(0.90-1.08)$ \\
\hline $\begin{array}{l}\text { Upper gastrointestinal (140-14I, } \\
\text { |43-146, 148-149) }\end{array}$ & 394 & 8 & 7.5 & $1.07(0.46-2.13)$ \\
\hline Larynx (16I) & 274 & 5 & 4.6 & $1.09(0.34-2.56)$ \\
\hline Salivary gland (142) & 109 & I & 2.5 & $0.39(0.0 \mathrm{I}-2.2 \mathrm{I})$ \\
\hline Nasopharynx (147) & 51 & I & 1.1 & $0.95(0.02-5.51)$ \\
\hline Oesophagus (I50) & 920 & 23 & 14.4 & $1.61(1.02-2.44)$ \\
\hline Stomach (15I) & 1565 & 20 & 23.8 & $0.84(0.5 \mathrm{I}-\mathrm{I} .30)$ \\
\hline Colon (153) & 1826 & 34 & 46.8 & $0.72(0.50-1.01)$ \\
\hline Rectum (154) & 1058 & 16 & 27.8 & $0.57(0.33-0.93)$ \\
\hline Liver ( 155$)$ & $34 I$ & 7 & 5.3 & $\mathrm{I} .33(0.53-2.78)$ \\
\hline Pancreas (I57) & 947 & 15 & 16.6 & $0.90(0.50-1.50)$ \\
\hline Lung (162) & 4693 & 88 & 75 & $1.18(0.94-1.45)$ \\
\hline Breast $(174,175)$ & 2659 & 80 & 79.4 & $1.01(0.80-1.26)$ \\
\hline Cervix $(180)$ & 262 & 10 & 8.6 & $1.17(0.55-2.18)$ \\
\hline Uterus (182) & 401 & 19 & 11.8 & $1.64(0.98-2.60)$ \\
\hline Ovary (183.0) & 473 & 15 & 14.3 & $\mathrm{I} .05(0.58-\mathrm{I} .75)$ \\
\hline Prostate (185) & 2536 & 19 & 25.0 & $0.76(0.46-1.19)$ \\
\hline Testis (186) & 168 & 4 & 3.1 & $\mathrm{I} .30(0.35-3.39)$ \\
\hline Kidney $(189.0,189.1)$ & 545 & 9 & 9.5 & $0.95(0.43-I .82)$ \\
\hline Bladder (188) & 1867 & 22 & 27.8 & $0.79(0.49-I .20)$ \\
\hline Skin $(172,173)$ & 2311 & 25 & 44.3 & $0.56(0.36-0.83)$ \\
\hline Malignant melanoma (I72) & 428 & 2 & 9.7 & $0.20(0.02-0.74)$ \\
\hline Other skin (I73) & 2093 & 23 & 37.6 & $0.61(0.38-0.91)$ \\
\hline Brain (malignant) $(191)$ & 506 & 7 & 9.5 & $0.74(0.29-1.53)$ \\
\hline Other nervous system (192) & 64 & I & 1.5 & $0.65(0.02-3.77)$ \\
\hline Thyroid (193) & 128 & 2 & 3 & $0.66(0.08-2.44)$ \\
\hline Bone (I70) & 187 & 3 & 3.6 & $0.83(0.17-2.47)$ \\
\hline $\begin{array}{l}\text { Non-Hodgkin's lymphoma } \\
\qquad(200,202)\end{array}$ & 911 & 15 & 17 & $0.88(0.49-1.46)$ \\
\hline Hodgkin's disease (20I) & 197 & 7 & 3.2 & $2.20(0.87-4.62)$ \\
\hline Multiple myeloma (203) & 495 & 7 & 7.8 & $0.89(0.36-1.85)$ \\
\hline Leukaemia (204-208) & 921 & 13 & 13.8 & $0.94(0.50-1.62)$ \\
\hline Lymphoid leukaemia (204) & 445 & 4 & 5.9 & $0.68(0.18-1.75)$ \\
\hline Myeloid leukaemia (205) & 462 & 7 & 7.5 & $0.93(0.37-1.93)$ \\
\hline Brain (benign) (225) & 291 & 10 & 7.6 & $\mathrm{I} .32(0.63-2.47)$ \\
\hline
\end{tabular}

I. ICD-9 (World Health Organization, 1978) codes for each cancer (equivalent codes were used for cases coded in other ICD revisions)

2. Conditions used in reference cohort, with Office of Population Censuses and Surveys (OPCS, 1975) code edition 3 for operations and ICD-9 code for diagnosis (with equivalent codes used for other coding editions): appendicecomy (OPCS $3,44 \mathrm{I}-444$; excluded from analysis of colorectal cancer), tonsillectomy/adenoidectomy (230-236), knee arthroplasty (812), hip arthroplasty $(810,811)$, squint (ICD-9 378), otitis externa, otitis media (380-382), haemorrhoids (455; excluded from the analyis of colorectal cancer), upper respiratory infections (460-466), deflected nasal septum, nasal polyp (470-47I), impacted tooth and other disorders of teeth (520-52I), inguinal hernia (550; excluded from the analysis of colorectal cancer, ingrowing toenail and other diseases of nail (703), sebaceous cyst (706.2), internal derangement of knee (717), bunion (727.I), selected fractures $(810-816,823-826)$, dislocations, sprains and strains $(830-839,840-848)$, superficial injury and confusion (910-919, 920-924).

3. Adjusted for gender, age in 5 -year bands, and time period in single calendar years. 
common in people with schizophrenia than in the reference cohort. Skin cancer was significantly less common in people with schizophrenia than in others (Table 2). The rate ratio for skin cancer was 0.56 $(P=0.004)$. Malignant melanoma and other skin cancers were, individually, less common in people with schizophrenia than in the reference cohort. The rate ratio for malignant melanoma was particularly low at $0.20(P=0.02)$. Cancer of the rectum was significantly lower in the schizophrenia cohort than in the comparison cohort, with a rate ratio of 0.57 (Table 2; $P=0.03$ ). Cancer of the colon was lower in the schizophrenia cohort than in the comparison cohort, but the result was not significant (rate ratio $0.72, P=0.06$ ).

The results presented here included patients of all ages when they had their first recorded admission for schizophrenia. We also analysed the data restricting the analyses to people whose first recorded admission for schizophrenia (or reference condition) was between the ages of 15 years and 64 years, reasoning that the identification of schizophrenia and of cancers is less likely to be reliable in the elderly than in the younger population. Apart from lowering statistical power, restriction of the age range in this way made no appreciable difference to the results.

\section{DISCUSSION}

\section{Background}

In 1909 the Commissioners in Lunacy for England and Wales wrote that cancer mortality might be relatively low 'among the insane' and that, if this were proved, it might 'have a bearing on its obscure etiology' (Commissioners in Lunacy for England and Wales, 1909). In that report, proportional mortality ratios were used to compare the death rates of those 'dwelling in insane asylums' with those of the general population. This was the basis for their suggestion that 'the insane' might be protected against cancer. Subsequent proportional mortality studies of individuals with schizophrenia supported this suggestion (reviewed by Scheflen, 1951). However, proportional mortality rates can be misleading. People with schizophrenia have high mortality rates for other, non-cancer, causes of death and, in particular, high mortality rates for unnatural causes of death (Herrman et al, 1983; Mortensen \& Juel, 1993). Accordingly, mortality rates for cancer, expressed as proportions of all causes of death, are low because death rates from other causes are high in people with schizophrenia. This point was made elegantly in the review of schizophrenia and cancer by Scheflen (1951).

Nonetheless, interest in the possible protective effect of schizophrenia persisted. The following three decades yielded a large number of studies (reviewed by Baldwin, 1979), generally of low statistical power, and further studies were published after Baldwin's review. For example, Craig \& Lin (1981) reported that the risk of lung cancer in people with schizophrenia may be low, despite the fact that high rates of smoking are well documented in patients with the latter disorder (Kelly \& McCreadie, 1999). Two large-scale, longterm studies in Denmark showed lower rates of several cancers, including lung cancer, in people with schizophrenia than in the general population (Mortensen, 1989, 1994). The first study followed a cohort of 6168 people with schizophrenia over a period of 27 years - by far the most substantial piece of work on this topic to date. It found a significantly reduced incidence of cancer overall in male patients as well as significant reductions in lung cancer and some other smoking-related cancers in both men and women (Mortensen, 1989). It found a small but significant elevation in the risk of breast cancer (rate ratio=1.19). The results were ascribed to the hospital environment experienced by psychiatric patients during the 1950 s and 1960s when, it was reported, smoking in the study population was said to have been restricted both by the patients' limited means and by regulations against smoking in Danish psychiatric hospitals (Mortensen, 1989). It was considered that a low pregnancy rate and reduced sexual activity could have accounted respectively for the increased risk of breast cancer and the finding of a non-significant reduced risk of cervical cancer among the female patients with schizophrenia (Mortensen, 1989).

A more recent, but less statistically powerful, cohort study was performed in Denmark to determine whether the earlier findings in an institutional environment, with restricted access to tobacco, were replicated in a more modern population of patients (Mortensen, 1994). The risk estimates generated by this study were less stable, since fewer malignancies were observed in the population. As before, cancer incidence overall was reduced in male patients. There was a non-significant reduction in lung cancer in men, based on six cases. The author concluded that the results of this second study did replicate those of the first and suggested that neuroleptic treatment might somehow be protective against cancer in people with schizophrenia (Mortensen, 1994).

\section{Findings in our study}

Our findings do not support the hypothesis that people with schizophrenia have a different overall risk of cancer from that of the general population.

\section{Cancers of the lung and oesophagus}

We did not find that lung cancer occurred less often in people with schizophrenia than in the general population. We found that the risk of lung cancer was non-significantly elevated and that the risk of cancer of the oesophagus was significantly elevated in people with schizophrenia, compared with the reference cohort. Smoking is the major risk factor for lung cancer and a high level of alcohol consumption is a risk factor for cancer of the oesophagus. The elevated rates of cancer of the lung and oesophagus may be attributable to smoking and alcohol consumption in the population with schizophrenia. We do not have data on smoking history or alcohol consumption. The research literature shows that people with schizophrenia are more likely to smoke than the general population (Kelly \& McCreadie, 1999). If there were much higher rates of smoking in the population with schizophrenia studied by us, we might have expected to see higher rates of lung cancer in these patients than we did. Thus, we cannot rule out the possibility that schizophrenia does protect against lung cancer to some extent. Nonetheless, because lung cancer rates in the schizophrenia cohort were high, our results indicate that it is unlikely that schizophrenia is associated with a major protective effect against lung cancer, and our results show no evidence of a general protective effect against other cancers.

\section{Breast cancer}

We found no elevation of breast cancer in our schizophrenia cohort, with a rate ratio of 1 . 


\section{Colorectal cancer}

We found a significantly low rate of rectal cancer. Dietary factors have a role in the aetiology of colorectal cancer, and there is evidence that there are differences in diet between people with schizophrenia and others (McCreadie, 2003), but a specific lowering of the risk of colorectal cancer is not a consistent feature of the literature on schizophrenia and cancer.

\section{Brain tumours}

There was no elevation of rates of brain tumours after a first diagnosis of schizophrenia. This shows that there was no evidence of any significant misdiagnosis of brain tumours as schizophrenia.

\section{Skin cancer}

We found that skin cancer occurred considerably less frequently in patients with schizophrenia than in the general population. There is not much published information about schizophrenia and skin cancer. However, a similar finding was reported from the second Danish recordlinkage study (Mortensen, 1994). The rate ratio in that study for malignant melanoma in people with schizophrenia, 0.14 , was similar to our ratio of 0.20 . Mortensen (1994) also reported a reduction in other skin cancers, similar to our risk estimate. There are several reasons why skin cancer rates may be lower than expected in people with schizophrenia. People with schizophrenia may spend less time in the sun than the general population. They may be less likely to seek medical advice than others. If doctors are less inclined to treat skin cancer in people with schizophrenia than in others, people with schizophrenia and skin cancer might be less likely to be admitted to hospital and therefore be selectively missed by our study method. Another possible explanation is that our findings might be due to chance: because we analysed 32 cancers, we would expect to find one or two statistically significant results by chance alone. However, with a prior hypothesis from the Danish record-linkage study that a low rate of skin cancer might be expected (Mortensen, 1994), and a significance level of 1 in $250(P=0.004)$ in our study, we are reluctant to accept chance as the most likely explanation. The low level of skin cancer is consistent with the hypothesis that sun exposure, perhaps through its role in vitamin D synthesis,

\section{CLINICAL IMPLICATIONS}

- There is a long-standing, unresolved debate about whether people with schizophrenia are protected against cancer. If they are, this might provide clues about aetiological mechanisms in schizophrenia.

- We found no evidence of a general deficit of cancer in people with schizophrenia.

- We found a deficit of skin cancer. A protective effect of solar radiation, perhaps mediated through vitamin D metabolism, in the development of schizophrenia is one possible explanation. A tendency of people with schizophrenia to avoid exposure to sunshine is another possibility.

\section{LIMITATIONS}

- The data-set was confined to people in contact with specialist hospital services.

- The cohorts were based on prevalent cases - the first recorded admission or day-case care for each person with each condition in the period covered by the data-set - rather than on incident cases.

- There was no information on behavioural factors such as smoking, diet or sun exposure.

MICHAEL J. GOLDACRE, FFPHM, Unit of Healthcare Epidemiology, Department of Public Health, University of Oxford, UK; LIANNE M. KURINA, PhD, Department of Health Studies, University of Chicago, Chicago, Illinois, USA; CLARE I.WOTTON, BSc, DAVID YEATES, PhD, VALERIE SEAGROATT, MSc, Unit of Healthcare Epidemiology, Department of Public Health, University of Oxford, UK

Correspondence: Professor Michael J. Goldacre, Unit of Health-Care Epidemiology, Department of Public Health, University of Oxford, Old Road Campus, Old Road, Oxford OX3 7LF, UK,

Tel: +44 (0)|865 226994; fax: +44 (0)1865 226993; e-mail: michael.goldacre@dphpc.ox.ac.uk

(First received 18 May 2004, final revision 7 December 2004, accepted 14 December 2004)

may have a protective role in the development of schizophrenia. Moskovitz (1978) suggested that vitamin $\mathrm{D}$ deficiency in the neonate might be a risk factor for the subsequent development of schizophrenia, and that this might be one explanation for the well-documented excess of winter births among people with schizophrenia. McGrath (1999) suggested that relative vitamin D deficiency could 'parsimoniously explain' diverse epidemiological features of schizophrenia such as the excess of winter births in people with schizophrenia and the increased rate of schizophrenia in dark-skinned migrants to climates with much more limited sunshine. If, in some people, solar radiation has a protective influence in the interaction of risk factors that lead to schizophrenia, skin cancer rates could be lower in people with schizophrenia than in others for that reason. There are other long-standing linked databases, for example in Scotland (Kendrick \& Clarke, 1993) and Western Australia (Holman et al, 1999), that could be used to determine whether the deficit of skin cancer in people with schizophrenia is a consistent finding. The methods used by van der Mei et al (2003), who made physical measurements of evidence of past skin exposure to solar radiation in people with multiple sclerosis, could be used to study the skin structure of people with and without schizophrenia.

\section{Strengths and weaknesses of the study}

The strengths of the study are that it provides information on large numbers of people in the schizophrenia cohort; and that, for the common cancers studied, there are large numbers of people with cancer in the cohort. The study design also has 
weaknesses. The database does not contain information on potential confounding or explanatory factors, in particular smoking, alcohol, diet and sun exposure. The database misses diseases that occurred in people who were not admitted to hospital, or who were admitted to a hospital outside its coverage, or who were admitted to hospital outside the period covered by the study. We have no information on the criteria used for the diagnosis of schizophrenia, other than the fact of its having been recorded as the clinical diagnosis on a hospital record. Although the diagnosis may sometimes be unreliable, it seems unlikely that its unreliability would be related to patients' risk of subsequent cancer. Accordingly, we think that diagnostic unreliability in some cases is unlikely either to create false associations with cancer or to mask completely any real associations. It is possible that important life-threatening diseases such as cancer are underdiagnosed, or undertreated, in people with schizophrenia. It is also theoretically possible that incidence rates for cancer could be underenumerated in schizophrenia because of competing causes of mortality for example, if undiagnosed cancers are 'overtaken' by deaths from other causes such as accidents. However, the fact that the overall rate ratio for cancer in our study is so close to 1 (Table 2 ) suggests that these are probably not major factors in the interpretation of our results. If they were, any real excess or deficit of cancers in the schizophrenia cohort would have to be precisely balanced by unidentified biases bringing the rate ratio back to 1 . This seems unlikely.

\section{Implications of our findings}

In conclusion, our data suggest that people with schizophrenia are not protected against cancer in general. There is no evidence of increased susceptibility to breast cancer. There is evidence that the risk of skin cancer may be low. As we are unable to take important lifestyle factors into account, such as smoking and exposure to solar radiation, our results should be regarded as suggestive rather than definitive.

\section{ACKNOWLEDGEMENTS}

Over many years, the linked data-files were built by Leicester Gill, Glenys Bettley and Myfanwy Griffith. The Unit of Healthcare Epidemiology is funded by the UK Department of Health's National Coordinating Centre for Research Capacity Development to analyse the linked data.

\section{REFERENCES}

Baldwin, J. A. (1979) Schizophrenia and physical disease. Psychological Medicine, 9, 611-618.

Basu, S. \& Dasgupta, P. S. (2000) Role of dopamine in malignant tumour growth. Endocrine, 12, 237-24I.

Breslow, N. E. \& Day, N. E. (1987) Statistical Methods in Cancer Research, vol. Il. The Design and Analysis of Cohort Studies. IARC Scientific Publication No. 82, pp 103-115. Lyon: International Agency for Research in Cancer

Carrillo, J. A. \& Benitez, J. (1999) Are antipsychotic drugs potentially chemopreventive agents for cancer? European Journal of Clinical Pharmacology, 55, 487-488.

Catts, V. S. \& Catts, S. V. (2000) Apoptosis and schizophrenia: is the tumour suppressor gene, p53, a candidate susceptibility gene? Schizophrenia Research, $4 \mathbf{I}$ $405-415$.

Commissioners in Lunacy for England and Wales (1909) Annual Report. London: HMSO

Craig, T. J. \& Lin, S. P. (1981) Cancer and mental illness. Comprehensive Psychiatry, 22, 404-410.

Goldacre, M., Kurina, L., Yeates, D., et al (2000) Use of large medical databases to study associations between diseases. Quarterly Journal of Medicine, 93. 669-675.

Herrman, H. E., Baldwin, J. A. \& Christie, D. (1983) A record-linkage study of mortality and general hospital discharge in patients diagnosed as schizophrenic. Psychological Medicine, 13, 581-593.

Holman, C. D., Bass, A. J., Rouse, I. L., et al (1999) Population-based linkage of health records in Western Australia: development of a health services research linked database. Australian and New Zealand journal of Public Health, 23, 453-459.

Kelly, C. \& McCreadie, R. G. (1999) Smoking habits, current symptoms, and premorbid characteristics of schizophrenic patients in Nithsdale, Scotland. American Journal of Psychiatry, 156, 175I-1757.

Kendrick, S. \& Clarke, J. (1993) The Scottish record linkage system. Health Bulletin, 5I, 72-79.

McCreadie, R. G. (2003) Diet, smoking and cardiovascular risk in people with schizophrenia: descriptive study. British Journal of Psychiatry, 183, 534-539.

McGrath, J. (1999) Hypothesis: is low prenatal vitamin $\mathrm{D}$ a risk-modifying factor for schizophrenia? Schizophrenia Research, 40, 173-177.

Mortensen, P. B. (1989) The incidence of cancer in schizophrenic patients. Journal of Epidemiology and Community Health, 43, 43-47.

Mortensen, P. B. (1994) The occurrence of cancer in first admitted schizophrenic patients. Schizophrenia Research, 12, 185-194.

Mortensen, P. B. \& Juel, K. (1993) Mortality and causes of death in first admitted schizophrenic patients. British Journal of Psychiatry, 163, 183-189.

Moskovitz, R. A. (1978) Seasonality in schizophrenia. Lancet, i, 664.

Office of Population Censuses and Surveys (1975) Classification of Surgical Operations (3rd revision). London: OPCS.

Scheflen, A. E. (195I) Malignant tumours in the institutionalized psychotic population. Archives of Neurology and Psychiatry, 66, 145-155.

Van der Mei, I. A. F., Ponsonby, A. L. \& Dwyer, T. (2003) Past exposure to sun, skin phenotype, and risk of multiple sclerosis: case-control study. BMJ, 327, 316-320.

World Health Organization (1978) International Statistical Classification of Diseases and Related Health Problems (ICD-9). Geneva: WHO.

Yovel, G., Sirota, P., Mazeh, D., et al (2000) Higher natural killer cell activity in schizophrenic patients: the impact of serum factors, medication, and smoking. Brain, Behavior, and Immunity, 14, 153-169. 